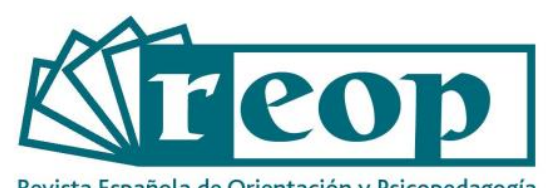

Revista Española de Orientación y Psicopedagogía

\title{
DESARROLLO DE LAS COMPETENCIAS EMOCIONALES DEL PROFESORADO DE SECUNDARIA MEDIANTE UNA INTERVENCIÓN INTEGRAL EN COACHING
}

\section{DEVELOPMENT OF EMOTIONAL COMPETENCES OF SECONDARY SCHOOL TEACHERS THROUGH A COMPREHENSIVE INTERVENTION IN COACHING}

\author{
Claudia Chianese ${ }^{1}$ \\ Universidad Ramón Llull. Facultad de Ciencias de la Educación y del Deporte. Departamento de \\ Pedagogía. Barcelona, España \\ Miquel Àngel Prats Fernández \\ Universidad Ramón Llull. Facultad de Ciencias de la Educación y del Deporte. Departamento de \\ Pedagogía. Barcelona, España
}

\section{RESUMEN}

La transformación que está viviendo la educación exige un cambio de rol para el docente, el tutor y el orientador y el desarrollo de sus competencias emocionales (CEmo), necesarias para facilitar una mejor orientación en el proceso de aprendizaje y crecimiento del alumnado. Para acompañarle en este reto es interesante aplicar procesos reflexivos, como el coaching, al ámbito educativo (CEd). El objetivo de nuestro estudio se centra en comprender los cambios percibidos por los miembros de un centro de secundaria en las CEmo de sus docentes, tras haber participado en un programa de CEd contextualizado. Se realizó un estudio de caso dividido en tres fases con un diseño integrado mixto. Nos centramos en la última fase, recogiendo datos cuantitativos, mediante el uso del cuestionario de desarrollo emocional CDE A-35, administrado a todo el claustro tras la intervención, y otros cualitativos, mediante entrevistas, focus group y

1 Correspondencia: Claudia Chianese. Correo-e: claudiac3@blanquerna.url.edu. web: Grupo de investigación PSITIC. http://recerca.blanquerna.edu/psitic/ 
encuestas, a distintos colectivos del centro. Los resultados cualitativos indican que los docentes perciben haber cambiado sus CEmo y los cuantitativos muestran un aumento en la media de casi todas las CEmo, sugiriendo que, con un programa más duradero, podrían ser significativos. En conclusión, el coaching facilita la reflexión del docente sobre su nuevo rol, como desarrollar sus CEmo, contribuyendo a la percepción de su mejora y de las de otros miembros del centro educativo. Asimismo, haber participado en estos programas y una sensibilidad previa al respecto, son claves para promover y consolidar cambios significativos en la función docente, tutorial y orientadora en un futuro.

Palabras clave: Coaching, inteligencia emocional, orientación escolar, formación del profesorado, desarrollo de competencias.

\section{ABSTRACT}

The transformation that education is going through demands a change of role for the teacher, tutor and counselor and the development of their emotional competencies (CEmo), necessary to facilitate better guidance to students throughout their learning process and growth of students. To accompany them in this challenge it is interesting to apply reflective processes, such as coaching, to the educational field (CEd). The objective of our study focuses on understanding the changes perceived by the members of a secondary school in the CEmo of their teachers, after having participated in a contextualized CEd program. A case study is divided into three phases with a mixed integrated design. We focus on the last phase, collecting quantitative data, using the CDE A-35 emotional development questionnaire, administered to the teaching staff, after the intervention, and other qualitative ones, through interviews, focus groups and surveys, to different groups at the school. The qualitative results indicate that teachers perceive that they changed their CEmo and the quantitative data show an increase in the average of almost all the CEmo and suggest that, with a more sustainable program, they could be significant. In conclusion, coaching facilitates the teacher's reflection on his/her new role, how to develop his/her CEmo, contributing to the perception of their improvement and that of other members of the school. Likewise, having participated in these programs and a prior sensitivity to them, are key to promoting and consolidating significant changes in the teaching, tutorial, and guidance function in the future.

Key Words: Coaching, emotional intelligence, school guidance, teacher training, skill development.

\section{Cómo citar este artículo:}

Chianese, C. y Prats, M.A. (2021). Desarrollo de las competencias emocionales del profesorado de secundaria mediante una intervención integral en coaching. Revista Española de Orientación y Psicopedagogía, 32(2), 110-131. https://doi.org/10.5944/reop.vol.32.num.2.2021.31282 


\section{Introducción}

“La emoción es el motor del aprendizaje” (F. Mora, 2017).

Instituciones como la OCDE (2016) o UNESCO (2016) consideran que, en el ámbito educativo, la selección de las competencias clave depende de lo que las sociedades valoren en cada momento y en cada contexto (Fernández Enguita, 2016; Pertegal, 2011). En el escenario actual, en el que las emociones han adquirido cada vez más importancia, dentro y fuera de los centros educativos, en el currículo de educación se ha procedido a la inclusión de las Competencias Emocionales ${ }^{2}$ (CEmo) desde el 2001 en el grupo de competencias básicas para la vida. Estas se consideran necesarias para que las personas puedan desarrollarse de manera integral y tengan éxito en su labor profesional y por ello el alumnado tiene que dominarlas al finalizar la escolaridad obligatoria (Riquelme, 2012).

Goleman (1996) afirma que, como la base de la inteligencia emocional es neurológica y el cerebro tiene una gran capacidad de modificarse con el aprendizaje, las diferencias en nuestra capacidad emocional pueden verse mejoradas con una intervención educativa adecuada (Riquelme, 2012).

En este sentido, la investigación ha demostrado que programas de formación específicos en Educación Emocional obtienen una mejora en el desarrollo de las CEmo, tanto en el profesorado de educación no superior en todas sus etapas formativas, desde infantil a secundaria (García Navarro, 2017; Pérez-Escoda et al., 2013; Ruzek et al., 2016; Torrijos, 2016; Torrijos et al. 2018) así como en el alumnado, desde la etapa de infantil (Gutiérrez-Lestón et al., 2018), hasta la universitaria (Pérez-Escoda et al., 2019), siendo por lo tanto los docentes, en todos los niveles, un impulso fundamental para cualquier mejora educativa.

Por ello se consideró necesario centrar la atención de este estudio sobre el profesorado, y, en primer lugar, sobre sus CEmo y cómo desarrollarlas.

Sin embargo, un modelo tradicional de enseñanza administrada de manera asimétrica que se ha consolidado en más de dos siglos de historia, durante los cuales el docente ha sido siempre la mayor fuente de conocimiento para el alumnado, en el siglo XXI, ya no funciona. Hay que pasar a una enseñanza-aprendizaje que logre hacer al estudiante responsable de su capacitación y al profesor un facilitador del mismo (Poblete, 2006) que le acompañe en esta transformación.

Por ello, en segundo lugar, en este estudio se consideró interesante centrar nuestra atención sobre el Coaching en Educación (CEd), un proceso que reúne los requisitos necesarios para poder apoyar al docente a realizar este cambio y desarrollar sus CEmo, fundamentales para generar unas buenas condiciones de aprendizaje y además similares a las que utiliza un coach (Albadalejo, 2010).

\footnotetext{
2 Definición de "Competencias emocionales del GROP": «El conjunto de conocimientos, capacidades, habilidades y actitudes necesarias para tomar consciencia, comprender y expresar, así como regular de forma apropiada los fenómenos emocionales». (Bisquerra, 2003).
} 


\section{Coaching}

El coaching es una modalidad de asesoramiento, que si bien deriva de varias disciplinas, muy distintas en sus teorías, si se quiere aplicar al campo de la educación, está influenciado principalmente por una evolución del counseling (Sánchez y Boronat, 2014). Sobre las bases de sus fundamentos, se sustentan unos principios: a) la importancia de la autonomía del sujeto y del autoaprendizaje; b) la conducta está condicionada por la peculiar visión del mundo que el sujeto se forma a lo largo de su vida; c) la necesidad de que coexista una alta motivación, responsabilidad hacia el cambio y deseo de transformación; d) la relevancia del proceso comunicativo que a través del lenguaje, de la verbalización de metas y del uso de preguntas poderosas, permite al individuo descubrirse y tender a actuar.

Durante los últimos 25 años se ha extendido este tipo de intervención a varios ámbitos y en la actualidad se pueden diferenciar distintos modelos de coaching y de escuelas. Ninguno se contradice y tienen puntos de unión, operando en todas las dimensiones del ser humano. Centramos nuestra atención sobre el CEd, encontrando múltiples definiciones. Al analizarlas observamos que lo que las une es el concepto de "aprendizaje" (Bécart y Ramírez, 2016; Grant, 2019; Salavert, 2015; Sánchez y Boronat, 2014), entendido como una forma nueva de aprender, más autónoma, posibilitando un mejor rendimiento, gracias al descubrimiento de las necesidades individuales de cada estudiante y ayudando a replantearse objetivos (Ozmen, 2019).

Hoy en día, el CEd y la educación, comparten el mismo propósito que es precisamente el de acompañar, más que instruir, siendo capaces de potenciar lo mejor de cada estudiante.

Estudios ya datados como el de Showers (1984) evidencian que el coaching no es un proceso nuevo en la formación del profesorado, considerándose como una estrategia eficaz en ella, tanto a nivel inicial, como permanente (Jiménez, 2012).

A estos, se suman estudios más recientes, tanto nacionales, como internacionales, más numerosos, que ponen de manifiesto el interés actual que suscita este planteamiento formativo y los efectos positivos de la aplicación del coaching sobre la educación. Estudios nacionales (López et al., 2018; Maestre et al., 2017; Rodríguez-Hidalgo et al., 2015) tienen como denominador común que el coaching ha permitido al profesorado alcanzar un mayor nivel de confianza, ayudándole en el desarrollo de la autonomía y responsabilidad del alumnado y modificando conductas para gestionar mejor los cambios.

Los estudios internacionales (Akiba y Wilkinson, 2016; Averill et. al., 2016; Bengo, 2016; Day et al., 2016; Rakap, 2017; Wood, 2016), se asemejan todos en cuanto al papel que asumen los docentes, que mejora una vez introducido el coaching en el aula, fortaleciendo su desarrollo profesional.

Se concluye que el coaching es un proceso eficaz alternativo a la pedagogía tradicional.

\section{Programas de CEd aplicados al desarrollo de CEmo}

Hay trabajos previos que sugieren que una intervención formativa en CEd puede incidir positivamente en el desarrollo de las CEmo de los docentes.

Uno es el de Obiols y Giner (2011), que realizaron un trabajo sobre profesores universitarios de dos facultades de la Universidad de Barcelona, en el cual se llevó a cabo una intervención formativa sobre un grupo de docentes, aportando un modelo de CEd estructurado en cuatro grandes bloques: inteligencia emocional, liderazgo, coaching y estrategias relacionales. Como resultados se expusieron los beneficios de introducir el rol de líder-docente-coach para facilitar el 
desarrollo del profesorado, y más en concreto sobre sus CEmo, las comunicativas relacionales y la de liderazgo docente.

Y otro de Rosa et al. (2015) que en su trabajo propusieron la introducción del coaching en una asignatura que se impartía para futuros docentes para desarrollar sus CEmo. Sus resultados confirman que el uso del coaching contribuye a la formación de profesionales reflexivos, capaces de manifestar sus emociones y pensamientos de forma respetuosa.

Sin embargo, hasta la fecha, no existen muchos estudios sobre la relevancia que puede tener el coaching a la hora de desarrollar las CEmo de unos docentes de secundaria y ninguno que haya explorado la posible influencia de una intervención integral de CEd sobre las percepciones de cambio en el desarrollo de las CEmo de los otros implicados en la comunidad educativa. Además, no hay ninguna intervención de CEd que antes de ser diseñada haya tenido en cuenta los factores que pueden facilitar o dificultar su realización, aplicabilidad y continuidad. Y, por último, tampoco hay constancia de programas que hayan complementado la formación, con un proceso de coaching individual dirigido a los docentes, así como de un acompañamiento llevado a cabo entre pares. Nuestro estudio quiere precisamente profundizar en estas necesidades, sirviendo el coaching como un proceso para el desarrollo de las CEmo del profesorado, y por lo tanto, facilitando unas buenas condiciones de aprendizaje para el alumnado.

\section{Objetivos de la investigación}

El objetivo general de nuestro estudio se centró en comprender los cambios percibidos por un claustro de docentes de secundaria en sus CEmo tras haber participado en una intervención integral de CEd contextualizado, así como las de otros miembros de la comunidad educativa.

Sus objetivos específicos fueron describir y comprender:

- Obj.1. Las percepciones de cambio de los docentes.

- Obj.2. Las percepciones de cambio del alumnado.

- Obj.3. Las percepciones de cambio de otros miembros de la comunidad educativa: equipo directivo y familias.

- Obj.4. Otros factores que influyeron en la percepción de cambio.

E identificar:

- Obj.5. Las diferencias entre los niveles de autopercepción de las CEmo docentes mediante el test CDE-A35 (pre-post.).

\section{Contexto y Programa de Intervención integral en CEd}

El programa de intervención integral de CEd contextualizado incluyó dos partes: (https://n9.cl/4senw)

a) Dirigida al $100 \%$ del claustro de docentes de la ESO (20 personas), de 15,5 horas de formación en coaching.

b) Dirigida al $25 \%$ del claustro, un grupo piloto (6 personas), de 9,5 horas, 4 de ellas de formación, 4 de un proceso con sesiones de coaching individuales, presenciales u online, impartidas por expertas y 1,5 de acompañamiento entre pares. Este colectivo participó tanto en la 
parte a) como en la b). Los integrantes fueron seleccionados de forma intencional por el equipo directivo en base a unos criterios específicos (ver Figura 1). Se decidió dirigir esta segunda parte (b) sólo a un grupo piloto por la imposibilidad de disponer de recursos necesarios para poderlo suministrar al claustro entero.

\section{Método}

\section{Diseño}

Para responder a los objetivos de la investigación se realizó un estudio de caso, desde un paradigma hermenéutico interpretativo, con enfoque principalmente cualitativo, usando un método mixto con diseño integrado (Creswell y Plano Clark, 2011), mediante el cual, para recoger la información, analizarla y evaluarla, se utilizó una combinación de procedimientos cuantitativos y cualitativos.

Tras diseñar y realizar la intervención de CEd, se procedió a analizar y evaluar el cambio percibido por los docentes sobre sus CEmo mediante entrevistas individuales focalizadas al grupo piloto y al equipo directivo, focus group a otros profesores y alumnado, por separado, y encuestas auto-administradas a familiares y al resto de docentes que quedaban por entrevistar. Se midió la percepción de sus CEmo mediante el mismo cuestionario usado en el pre-test, suministrado a los docentes (post-test). Se procedieron a analizar los datos según su tipología mediante análisis temática y descriptiva inferencial de forma separada. Los datos cuantitativos sirvieron principalmente para alcanzar dos objetivos: primero para que cada docente tomase consciencia sobre su nivel de CEmo y supiese hacia dónde dirigir su interés de desarrollo durante la intervención y, segundo, para dotar de una mayor contundencia la percepción de cambios observados a nivel cualitativo.

\section{Contexto y participantes}

La escuela escogida como caso contextual fue un centro de secundaria en Barcelona, Cataluña ${ }^{3}$.

El caso fue seleccionado intencionalmente, por un lado, por un tema de accesibilidad, dado que años atrás se había realizado un programa formativo con el claustro de primaria, cuya satisfacción había sido elevada, y por otro, por una gran afinidad entre la visión y los valores del centro y la filosofía que mueve el CEd.

La elección de los participantes del centro fue un muestreo no probabilístico intencional siguiendo determinados criterios (Figura 1).

Por un lado, fue seleccionado el claustro completo de secundaria que estaba compuesto por 20 docentes en activo (inicialmente en el pre-test fueron 23, pero posteriormente se perdieron 3

${ }^{3}$ http://www.fedac.cat/amilcar.php 
sujetos por baja por enfermedad) que impartieron docencia durante el curso 2018-2019 en varias especialidades. Por otro lado, intervinieron otros miembros del centro.

\section{Figura 1}

Características de los participantes del estudio

\begin{tabular}{|c|c|c|c|c|c|c|}
\hline Colectivo & Hombres & Mujeres & $\begin{array}{l}\text { Edad } \\
\text { Media } \\
\text { (Años) }\end{array}$ & $\begin{array}{l}\text { Criterios } \\
\text { selección }\end{array}$ & $\begin{array}{l}\text { Procedimiento } \\
\text { selección }\end{array}$ & $\begin{array}{c}\text { Perfil } \\
\text { Participante } \\
\text { (Formación } \\
\text { Y } \\
\text { Profesión) } \\
\end{array}$ \\
\hline $\begin{array}{l}\text { Equipo } \\
\text { Directivo } \\
\text { (ED) }\end{array}$ & 1 & 1 & 47,5 & Miembro ED de la ESO & $\begin{array}{c}\text { Todo el ED de } \\
\text { ESO }\end{array}$ & Letras \\
\hline Docentes & 6 & 17 & 47,4 & Docente de 10 a $4^{\circ}$ ESO & $\begin{array}{c}\text { Todo el claustro } \\
\text { de ESO }\end{array}$ & $\begin{array}{l}-12 \text { Letras } \\
-8 \text { Ciencias } \\
\text { Mat. } \\
\text { (1 Doctor) } \\
-3 \text { Ciencias } \\
\text { Deporte }\end{array}$ \\
\hline $\begin{array}{c}\text { Grupo } \\
\text { piloto } \\
\text { docentes }\end{array}$ & 2 & 4 & 44,3 & $\begin{array}{c}\text {-Docente de 10 a 40 } \\
\text { ESO } \\
\text {-25\% claustro } \\
\text { participante } \\
\text {-Ambos géneros parte } \\
\text { proporcional } \\
\text {-Mayor y menor } \\
\text { Antigüedad centro } \\
\text {-Algunos tutores ESO } \\
\text {-Algunos miembros ED } \\
\text {-Interesados y } \\
\text { motivados hacía } \\
\text { innovación educativa } \\
\text {-Comprometidos }\end{array}$ & \multirow{2}{*}{$\begin{array}{l}\text { La Dirección } \\
\text { pedagógica de } \\
\text { ESO eligió según } \\
\text { criterios } \\
\text { indicados }\end{array}$} & $\begin{array}{c}-3 \text { Letras } \\
-3 \text { Ciencias } \\
\text { Mat. }\end{array}$ \\
\hline Alumnado & 4 & 6 & 14,1 & $\begin{array}{c}\text {-Cursar 2\%/3\%/40 ESO } \\
\text {-Participativo } \\
\text {-Maduro } \\
\text {-Ganas de aportar y } \\
\text { criticar } \\
\text { constructivamente }\end{array}$ & & 20 a $40 \mathrm{ESO}$ \\
\hline Padres & 2 & 4 & 50 & $\begin{array}{c}\text {-Padre/madre del } \\
\text { alumnado de 10 a 40 } \\
\text { ESO } \\
\text { - Implicados y } \\
\text { participativos } \\
\text {-Sensibles hacía temas } \\
\text { de innovación en } \\
\text { educación } \\
\text { - Con ganas de aportar } \\
\text { y criticar } \\
\text { constructivamente } \\
\text {-Conocedores del } \\
\text { claustro } \\
\end{array}$ & $\begin{array}{c}\text { El Director } \\
\text { General envió } \\
\text { un correo } \\
\text { mediante } \\
\text { “Clickedu” a las } \\
\text { familias según } \\
\text { criterios } \\
\text { indicados y } \\
\text { participaron las } \\
\text { que } \\
\text { respondieron }\end{array}$ & $\begin{array}{c}-3 \text { Lic. } \\
\text { Ciencias } \\
(2 \\
\text { ingeniería; } \\
1 \\
\text { enfermería) } \\
-3 \text { Diplo. } \\
(1 \\
\text { enfermería; } \\
2 \text { otro) }\end{array}$ \\
\hline
\end{tabular}

Fuente: Elaboración Propia

En todo momento se respetó la confidencialidad de los datos y los estándares éticos requeridos para trabajar con personas, haciendo firmar a todos los implicados un documento de consentimiento informado para el conocimiento de la investigación y el uso de sus datos, así como para la grabación de audios. 
En el caso de menores, fueron los padres quienes tuvieron el derecho de autorizar o no la participación de sus hijos en el estudio.

\section{Instrumentos}

En el trabajo de campo se utilizaron varias herramientas de recogida de información administradas de forma distinta según el colectivo y validadas por unos jueces expertos (https://n9.cl/8caq).

Para las entrevistas, los focus group y las encuestas se utilizó una guía que abordaba la percepción y opinión de los participantes sobre varios aspectos considerados de interés para comprender los cambios tras la intervención de CEd.

Mediante el cuestionario de desarrollo emocional CDE A-35, elaborado por el GROP (PérezEscoda et al., 2010) se midieron los niveles de CEmo de los docentes, antes de la intervención y después (pre-test y post-test). Este cuestionario está compuesto de 35 ítems distribuidos en cinco CEmo y ofrece una puntuación global de la CEmo y otras parciales, para cada una de las cinco dimensiones (Figura 2). Se trata de una escala tipo Likert de 11 puntos con un coeficiente de fiabilidad medido mediante el alfa de Cronbach, de 0,92 para la escala completa y superior a 0,69 para cada una de las cinco competencias.

\section{Figura 2}

\section{Modelo Competencias emocionales}

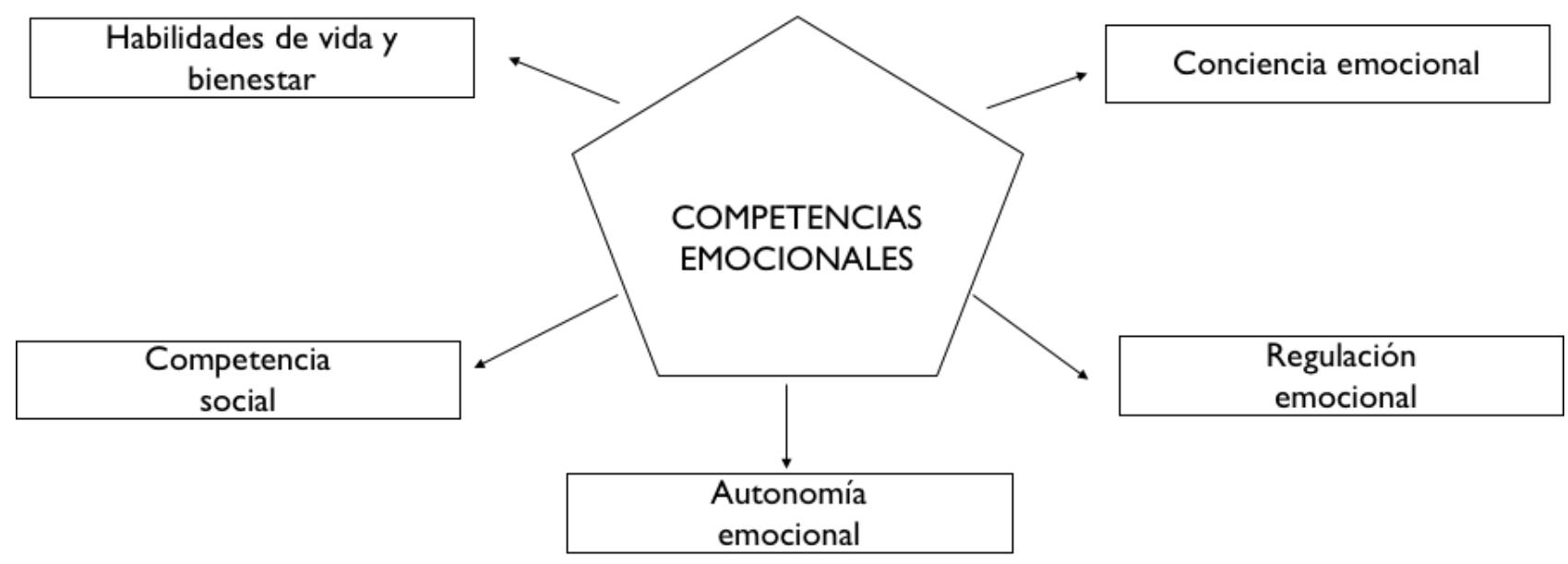

Fuente: Bisquerra y Pérez-Escoda, 2007

\section{Procedimiento de recogida de información}

Una vez terminado el programa, para recoger la información mediante las entrevistas y los focus group se concertó una fecha con cada participante durante el mes de junio de 2019.

La duración de las entrevistas y de los focus group varió y en la mayoría de los casos fue entre 20 y 30 minutos. 
Fue la misma investigadora principal quien organizó el proceso de envío y cumplimentación de los cuestionarios CDE A-35, así como de las encuestas, fijando un plazo para aplicar los mismos.

\section{Procedimiento de análisis de datos}

Para el tratamiento de los datos obtenidos mediante entrevistas, focus group y encuestas se realizó un análisis temático que se llevó a cabo siguiendo las 6 fases descritas por Braun y Clarke (2006) en su investigación:

- 1. Familiarización con los datos: Mientras se trascribieron textualmente las entrevistas y los focus group, se tuvo la oportunidad de leer múltiples veces la información y empezar a apuntar unas primeras ideas.

- 2. Generación de códigos iniciales: Mediante el uso del software informático Atlas.ti, versión 8.4.4, se procedió a una primera codificación de todas las respuestas de los participantes. Se identificaron las características interesantes de los datos de manera sistemática en todo su conjunto, recopilando datos relevantes para cada código, que se delimitaron de forma independiente según las unidades de información y los temas significativos.

- 3. Búsqueda de temas: Tras haber coleccionado una primera larga lista de múltiples códigos (casi 595) se pasó a analizarlos, fusionarlos y combinarlos entre ellos para formar temas y sub-temas potenciales. Durante este proceso se usaron redes para ayudar a representar visualmente tal cantidad de códigos. Se identificaron temas que se repetían en varios colectivos, sus puntos comunes y sus diferencias, empezando a crear grandes grupos de códigos que más adelante pasaron a ser nuestras principales dimensiones. Se finalizó esta fase con un listado más reducido de temas potenciales (13) y subtemas (105), empezando a dar significado a cada uno de ellos individualmente.

- 4. Revisión de temas: Se refinaron los datos de la fase anterior, diferenciando dos niveles de revisión. Un primer nivel, para ver si todos los códigos identificados en un mismo tema llegaban a formar algo coherente, relacionándose entre ellos, y un segundo nivel, donde se siguió un proceso similar, pero en este caso para el conjunto de los datos completos, generando un "mapa" temático del análisis.

- 5. Definición y nombramiento de temas (dimensiones): Se definió cada tema, asignándole un nombre claro, identificando finalmente 6 dimensiones, y varias categorías (Figura 3).

- 6. Producción de la relación: Se finalizó el análisis cuando se respondió a la pregunta de investigación inicial y sus objetivos específicos mediante las dimensiones identificadas, pasando a redactar el informe.

Por otra parte, para analizar los datos cuantitativos obtenidos mediante el CDE A-35, se almacenaron en una matriz de datos y se realizó una codificación de las variables en una hoja de cálculo mediante el software M.S. Excel del paquete Office versión 2016.

Se realizaron los siguientes análisis estadísticos: a) descriptivos univariados de todas las variables implicadas (de las 5 CEmo y del total de las CEmo en el pre y en el post); b) pruebas de normalidad y pruebas de contrastes de hipótesis paramétricas mediante el test de hipótesis Shapiro-Wilk, disponiendo de una muestra de menos de 50 sujetos; c) descriptivos bivariados para explorar la relación entre las variables, adecuadas al tipo de datos; d) inferenciales (t-Student), para determinar la significatividad de las diferencias encontradas y e) la $d$ de Cohen (effect size), 
para valorar la magnitud del cambio ocurrido en el post-test una vez finalizado el programa. Para todos los estadísticos se usó el programa informático IBM SPSS Statistics en su versión 20.0.0.

\section{Figura 3}

Mapa de dimensiones y categorías finales

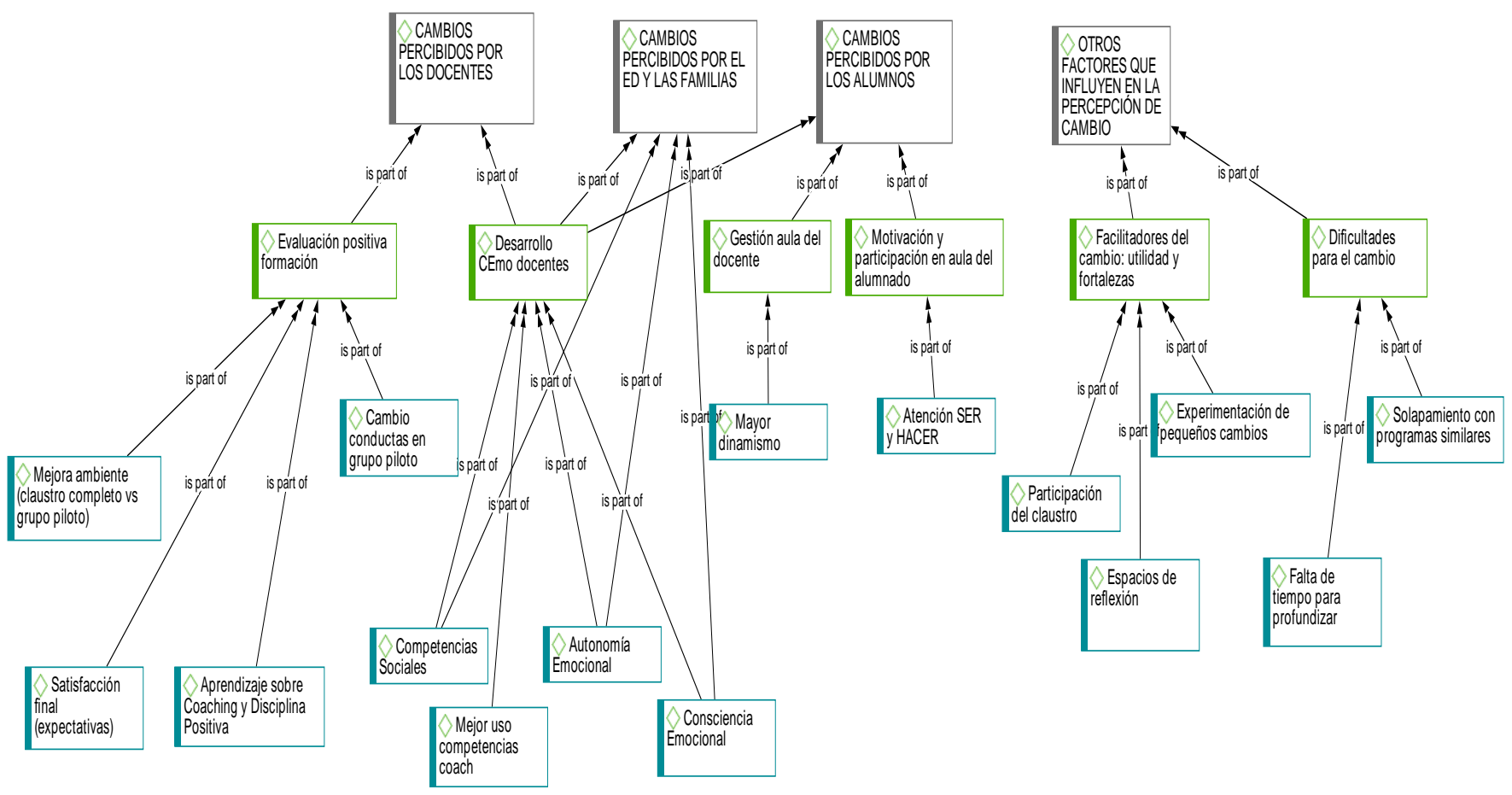

Fuente: Elaboración propia mediante el soporte del programa informático Atlas.ti (versión 8.4.4)

Finalmente, para asegurar la calidad y contraste de los datos y dar una mayor credibilidad a la investigación (Sandín, 2003), se confrontó la información mediante una triangulación de los mismos provenientes de las distintas técnicas utilizadas, así como de los grupos informantes.

\section{Resultados}

Mediante el análisis temático se categorizaron 6 dimensiones percibidas como cambios tras la intervención en CEd: una se observó en todos los grupos entrevistados, la mejora de las CEmo de los docentes y las otras cinco fueron distintas, según el colectivo implicado. 


\section{Obj. 1: Las percepciones de cambio de los docentes}

Las percepciones de cambio citadas por el claustro fueron: por un lado, la mejora de sus CEmo (dimensión 1), destacando sobre todo algunas de entre ellas y, por otro, aspectos referentes a la evaluación positiva de la formación (dimensión 2). Entre estos últimos se mencionaron: a) la satisfacción al finalizar el programa, b) el aprendizaje nuevo adquirido mediante la formación, c) los cambios percibidos en las conductas de algunos docentes del grupo piloto y d) la mejora del ambiente entre los profesores, sobre todo referente a las diferencias entre el clima del claustro completo y el del piloto (Cuadro 1).

\section{Cuadro 1}

Dimensiones percibidas por los docentes tras la intervención integral en CEd

\begin{tabular}{ll}
\hline \multicolumn{1}{c}{ Cambios percibidos por los docentes } \\
\hline Dimensiones & Categorías \\
\hline Desarrollo CEmo docentes & Autonomía Emocional \\
& Competencias Sociales \\
& Mejor uso competencias docente-coach \\
Evaluación positiva formación & Satisfacción final (expectativas) \\
& Aprendizaje sobre coaching y disciplina positiva \\
& Cambio conductas grupo piloto \\
& Mejora ambiente (claustro completo vs grupo piloto) \\
\end{tabular}

Fuente: Elaboración propia

\section{Desarrollo de sus CEmo (dimensión 1)}

Los profesores destacaron diferentes aspectos que habían mejorado en cada CEmo, siendo la Autonomía Emocional $(\mathrm{AE})$ entre las más mencionadas. En concreto, percibieron una mayor autoestima y capacidad de auto-motivarse, que fueron las dos componentes que comentaron en la mayoría de casos, seguidas por más autonomía, capacidad de resiliencia frente a las adversidades, así como el saber delegar y tomar decisiones.

M.S.: Yo quizás a ser más consciente que necesito la aceptación (...), o el reconocimiento de los iguales, de mis compañeros y quizás intentar que no me afecte tanto cuando no hay este reconocimiento...ser más consciente que (...) no es necesario siempre que se muestre.

(Focus group con docentes).

Conjuntamente a la $\mathrm{AE}$, la otra CEmo más nombrada, fue la Competencia Social (CS), que varios profesores entrevistados percibieron haber mejorado. Mencionaron que se atrevían más a dar feedback sobre lo que pensaban, aunque no fuera siempre positivo, a realizar una mayor práctica de la escucha empática con compañeros y con estudiantes y a tener más atención para prevenir y solucionar conflictos. 
A.S.: Yo en la competencia social, en la capacidad de gestionar situaciones emocionales y la prevención y solución de conflictos. Veo que aquí, pues he hecho un pequeño avance. De entenderme y entender. Que era la que en mi caso salía más baja y la que a nivel de trabajo personal (...) he trabajado. (Entrevista con grupo piloto docentes ).

Por las otras CEmo, unos pocos docentes entrevistados, mencionaron haber desarrollado la Consciencia Emocional (CE), considerándose sobre todo más conscientes de tener que realizar un cambio. Algunos profesores destacaron haber desarrollado la Regulación Emocional (RE), notándose más capaces a la hora de gestionar ciertas situaciones, pensando más antes de actuar y utilizando más los silencios frente a actitudes negativas. Solo un caso destacó haber desarrollado la Competencia del Bienestar y de la Vida (CBV), diciendo que se había cuidado más y había disfrutado más de las cosas.

B.P.: En cuanto a cosas que he mejorado ...quizás en el tema de la regulación, de pensar antes. (Focus group docentes).

M.M.: Porque también tengo que cuidarme a mí, que va un poco relacionado con el bienestar y en la vida, quiero hacer el cambio porque me apetece como propuse disfrutar también de las cosas. (Focus group docentes).

Los profesores mencionaron haber mejorado el uso de sus competencias como "docentecoach", siendo precisamente las que coinciden en su mayoría con las CEmo: como realizar una mayor escucha empática hacía el alumnado y a sus compañeros, el dar y recibir feedback a y de parte de otros docentes (CS), y además, el no juzgar, el saber decir que no, el fomentar la autonomía del alumnado, el diferenciar lo que es un aliento de lo que es un elogio, el usar las preguntas poderosas y las metas erradas.

A.G.: Yo lo he intentado llevar a la práctica en las tutorías individualizadas (...) sobre todo (...) lo de la escucha empática, hacerles preguntas, para que ellos encontraran una respuesta, un poquito las técnicas. (Focus group docentes).

\section{Evaluación positiva de la formación (dimensión 2)}

En la segunda dimensión confluyeron los cuatro niveles de evaluación de una acción formativa citados por Kirkpatrick y Kirkpatrick (2006), todos valorados positivamente.

a) La primera categoría que mencionaron fue la satisfacción hacia al programa, superando las expectativas iniciales, que en general no eran muy altas.

M.L.: Yo diría que las ha superado sí porque realmente (...) cuando empiezas una formación (...) dices, vale voy a aprender, pero no piensas que te vaya a afectar a nivel personal (...) que te va a llegar tanto. (Entrevista con grupo piloto docentes ).

b) En segundo lugar, consideraron tener un mayor conocimiento sobre la temática del CEd, dado que una parte de su nuevo aprendizaje se refería al modelo propuesto de coaching, el Ser Más (Valderrama, 2015), y otra, a conceptos de la disciplina positiva (Nelsen et al., 2013). Todos los profesores valoraron mucho las nuevas dinámicas aprendidas en clase.

N.G.: Yo creo que todo lo que he visto en la formación, incluidos los vídeos o las reflexiones. (Entrevista con grupo piloto docentes ). 
c) En tercer lugar, hablaron de un cambio de conducta muy positivo observado en algunos compañeros del grupo piloto, que habían trabajado con más profundidad.

E.C.: He visto alguna compañera que sí que ha cambiado...que le ha sido como positivo. (Focus group docentes).

d) La cuarta categoría fue la mejora en el ambiente del mismo claustro, dado que en general el clima percibido en el grupo completo fue muy bueno, participativo y dinámico, superando las expectativas iniciales. En concreto, la sensación de las personas que formaron parte del grupo piloto fue de haber generado entre ellas un ambiente de mayor confianza con respecto al del grupo entero, ya positivo, dado que se pudieron mostrar más tal y como eran, en su vulnerabilidad. Todo esto fue facilitado por ser un grupo más reducido y, por lo tanto, tener más confianza y comodidad a la hora de participar y abrirse entre ellos.

D.F.: Claro evidentemente es distinto estar 25 que 5 ¿no? (...) te sientes más ...no más a gusto, porque yo también he estado muy a gusto en el mayor, pero a ser un grupo reducido (...) tienes más confianza. Quizás. (Entrevista con grupo piloto docentes ).

A.T.: En el grupo pequeño (...) hay un ambiente más distendido, la gente se muestra más como es (...) más vulnerable. Pero porque también el grupo, entre nosotros, nos avenimos. (Entrevista con grupo piloto docentes).

\section{Obj. 2: Las percepciones de cambio en el alumnado}

Se percibió que el alumnado que había sido previamente informado por parte de sus profesores del programa que estaban realizando, se mostraba más sensible a notar cambios en las CEmo de sus docentes y por ello se observaron diferencias en la información recogida en los focus group, según los profesores que habían tenido.

S.: A nosotros nuestra tutora nos explicó un poco que ahora los profesores estaban haciendo una formación por todo esto y nos hizo hacer una encuesta para ver si había cambiado algo. (Alumno de 4ํㅡㄹ $\mathrm{ESO}$, Focus group con estudiantes).

Aunque fue más difícil identificar temas comunes, entre estos estudiantes más sensibilizados se diferenciaron dos nuevas dimensiones: una distinta gestión del aula por parte del docente y una mayor motivación y participación en clase por parte del mismo alumnado, aparte de volver a mencionar el desarrollo de las CEmo de sus profesores (Cuadro 2).

\section{Cuadro 2}

Dimensiones percibidas por el alumnado tras la intervención integral en CEd

Cambios percibidos por el alumnado

\begin{tabular}{lc}
\hline \multicolumn{1}{c}{ Dimensiones } & Categorías \\
\hline Desarrollo CEmo docentes & \\
Gestión aula del docente & Mayor dinamismo \\
$\begin{array}{l}\text { Motivación y participación en aula } \\
\text { del alumnado }\end{array}$ & Atención SER y HACER \\
\hline Fuente: Elaboración propia
\end{tabular}

Fuente: Elaboración propia 


\section{Desarrollo de las CEmo docentes (dimensión 1)}

El alumnado consideró que, tras la intervención, sus educadores compartían más información con ellos (CS), empatizaban más (CS), se enfadaban menos (RE), siendo más amables y teniendo más disposición a la hora de hablar (CS).

\section{S.: Es como que soportan más...el comportamiento o sea antes se enfadaban más. (Focus} group con estudiantes).

L.: Yo M.R. ...aquí pone "compartir emociones" ...que se le nota mucho cuando le pasa algo y a veces nos lo intenta transmitir para que también entendamos su situación como profesora. (Entrevista equipo directivo).

R.: Actitud positiva y automotivación...el T. (Alumnado $2^{\text {a }}$ ESO, focus group con estudiantes).

Gestión del aula por parte del docente (dimensión 3)

Además, los estudiantes notaron diferencias en la gestión del aula de parte de sus profesores, que en su opinión utilizaban actividades más dinámicas y menos teoría (CS).

M.: Sí por ejemplo en la clase de biología, hemos hecho más trabajos y menos temario (...) es más interactivo...es guay. (Alumna 4ํㅡㄹ ESO, focus group con estudiantes).

\section{Motivación y participación del alumnado en el aula (dimensión 4)}

Y todo esto parece que influyó positivamente, aunque no se pueda hablar por todo el alumnado entrevistado, sobre su mayor motivación y participación en clase.

L.: Sí porque también ayuda por ejemplo si ves a un profesor que es más empático (...) a que no tengas tanta vergüenza a hablar en alto. (Alumno $2^{\circ} \mathrm{ESO}$, focus group con estudiantes).

Obj.3: Percepción de cambios de otros miembros de la comunidad educativa: equipo directivo (ED) y familias

En el caso del ED y de los familiares, resultó más complejo hablar de una percepción de mejora, por el número limitado de personas de este colectivo y la discrepancia de percepciones identificadas en un mismo grupo.

Como en el caso del alumnado, la sensibilidad y el conocimiento previo del ED influyeron positivamente en la percepción de cambios, generando un efecto de casi "contagio" como comentó uno de los miembros del ED:

E.E.: ... Tuve un momento así un poco de presión y un poco de agobio también y (...) una persona que hacía coaching me lo hizo a mí y me ayudó muchísimo porque fue un momento de poder expresar las emociones y entonces yo recibir un apoyo y una ayuda.

...Fue muy interesante...para mí fue como esta cadena de favores que (...) llegó a mí y a mí también que es algo que me gusta y estoy familiarizada, me despertó otra vez el poder poner en práctica ese tipo de habilidades sociales con los demás, es como que creo que es algo que se contagia y creo que ha habido contagio, quizá en el círculo. (Entrevista equipo directivo). 


\section{Desarrollo de las CEmo docentes (dimensión 1)}

Solo un miembro del ED percibió algún tipo de cambio y nuevamente como dimensión se volvió a identificar el desarrollo de las CEmo docentes (Cuadro 3).

\section{Cuadro 3}

Dimensión percibida por el ED y las Familias tras la intervención integral en CEd

\begin{tabular}{cl}
\hline \multicolumn{1}{c}{ Cambios percibidos por el ED y las familias } \\
\hline Dimensiones & \multicolumn{1}{c}{ Categorías } \\
\hline Desarrollo CEmo docentes & Competencias Sociales \\
& Autonomía Emocional \\
& Consciencia Emocional \\
\hline
\end{tabular}

Fuente: Elaboración propia

Notó una mejora concretamente en las CS del claustro. Percibió más empatía entre los docentes, una mayor implicación y participación en personas que anteriormente no solían exponerse mucho, así como más iniciativa $(A E)$ y CE.

E.E.: Gente de claustro sobre todo de secundaria que a veces está más pasiva (...). Si que hemos visto que la gente estaba participativa y más dada a meterse. Quizás (...) en autonomía emocional, respeto a tener iniciativas y proponer cosas, (...) también al equipo directivo, soluciones o alternativas. (Entrevista equipo directivo).

En el caso de los familiares, fue casi imposible observar una percepción de cambio, por el poco trato con los docentes de sus hijos durante la realización del programa, aspecto que quedó reflejado en la devolución de solo 2 de las 6 encuestas enviadas a los padres. Igualmente, una familia mencionó una mejora en cuanto a la CS:

M.S.: La comunicación es más directa, facilitan las entrevistas y si es necesario por teléfono, ante algún problema (...) ha habido una buena respuesta por parte de los docentes: nos hemos sentido escuchados y se han buscado soluciones conjuntas. (Encuesta, madre).

\section{Obj. 4: Otros factores que influyen en la percepción de cambio}

Por último, se percibieron dos dimensiones en varios colectivos. Unos factores facilitadores, y otros que dificultaron que se realizasen las percepciones de cambio anteriormente descritas (Cuadro 4). 


\section{Cuadro 4}

Otras dimensiones percibidas tras la intervención formativa en CEd

\begin{tabular}{ll}
\hline \multicolumn{1}{c}{ Otros factores que influyen en la percepción de cambio } \\
\hline \multicolumn{1}{c}{ Dimensiones } & \multicolumn{1}{c}{ Categorías } \\
\hline $\begin{array}{l}\text { Facilitadores del cambio: utilidad y } \\
\text { fortalezas }\end{array}$ & $\begin{array}{l}\text { Participación del claustro } \\
\text { Espacios de reflexión } \\
\text { Experimentación de pequeños } \\
\text { cambios }\end{array}$ \\
Dificultades para el cambio & $\begin{array}{l}\text { Falta tiempo para profundizar } \\
\text { Solapamiento con programas } \\
\text { similares }\end{array}$ \\
\hline
\end{tabular}

Fuente: Elaboración propia

Facilitadores del cambio: utilidad y fortalezas (dimensión 5)

Entre los puntos fuertes y de utilidad se mencionaron la gran participación del claustro en la intervención, el haber tenido espacios para reflexionar y la posibilidad de experimentar de forma práctica cambios de conducta.

N.E.: Como temas positivos (...) he visto implicación por parte del equipo docente, (...) he visto ganas e ilusión que normalmente no suelo detectarlo cuando es una formación de otra tipología (...) han venido en horas que no les tocaba alguna persona para hablar contigo o para hacer algún comentario con el grupo, así que esto también (...) lo valoro de manera muy muy especial. (Entrevista equipo directivo).

\section{Dificultades para el cambio (dimensión 6)}

Entre las dificultades para facilitar el cambio, se nombraron: la falta de tiempo para profundizar y la de una estructura común entre formaciones similares realizadas en paralelo en el centro y nuestro programa.

E.C.: En este sentido ha sido como corto (...) yo he tenido la sensación que de cara a profundizar con el tema alumnos, allí me ha faltado. (Focus group docentes).

M.D.: Yo un poco desde el desconocimiento, pero me preocupa que superpongamos diferentes cosas simultáneamente, sin delimitar claramente (...) tenemos el líder en mí en marcha, coaching... habrán otros cursos (...), irá creciendo en cursos... las dos cosas son complementarias, pero de momento están como islas (...) ... creo que no hay una visión global de cómo encaja todo esto, que sean islas que se van haciendo y que al final también se van sobreponiendo sobre otras, hablando de lo mismo con lenguajes diferentes. (Focus group docentes). 
Obj. 5: Diferencias entre los niveles de autopercepción de las CEmo docentes mediante el CDE A-35 (pre-post)

Como indica la Tabla 1:

- a. En la AE observamos una mejora de más de un punto en la media del post $(5,68)$ con respecto a la del pre $(4,67)$ y en general, en casi todas las CEmo del post, notamos un ligero aumento en positivo.

- b. Vemos una falta de diferencias significativas entre las puntuaciones del pre y del post de las variables medidas (la $t$ de Student.). El valor obtenido en la competencia de $\mathrm{AE}$ es de un 0,068 de significatividad, siendo la CEmo que más se acerca a ser significativa estadísticamente. Esta misma en el pre-test, obtuvo el valor medio más bajo entre las 5 . El resto de las competencias obtienen todas niveles mayores de 0,05, por lo tanto, no significativas.

- c. Observamos un valor entre trivial y small (Hopkins, 2009) de la $d$ de Cohen (effect size) en la AE y en el Total de CEmo (Lenhard y Lenhard, 2016). En el caso de la AE es de 0,49, y en el total CEmo, de 0,25; en todas las otras CEmo el efecto es trivial (excepto la CE que es negativo).

Tabla 1

Valores estadísticos obtenidos comparando el pre y el post test de la medición de las CEmo del claustro mediante el uso del test CDE A-35

\begin{tabular}{llll}
\hline & Pre (mean-sd) & Post (mean-sd) & $\begin{array}{l}\text { Valor-p } \\
\text { (d-cohen) }\end{array}$ \\
\hline $\begin{array}{l}\text { Consciencia } \\
\text { emocional (CE) }\end{array}$ & $7,69(1,07)$ & $7,67(0,84)$ & $0,927(-0,02)$ \\
\hline $\begin{array}{l}\text { Regulación } \\
\text { emocional (RE) }\end{array}$ & $6,73(1,19)$ & $6,97(1,35)$ & $0,344(0,19)$ \\
\hline $\begin{array}{l}\text { Autonomía } \\
\text { emocional (AE) }\end{array}$ & $4,67(1,63)$ & $5,68(1,21)$ & $\mathbf{0 , 0 6 8 ( 0 , 4 9 )}$ \\
\hline $\begin{array}{l}\text { Competencias } \\
\text { sociales (CS) }\end{array}$ & $6,49(1,68)$ & $6,53(1,76)$ & $0,556(0,02)$ \\
\hline $\begin{array}{l}\text { Competencias de la } \\
\text { vida (CV) }\end{array}$ & $7,91(0,84)$ & $8,07(0,95)$ & $0,327(0,18)$ \\
\hline Total: CEMO & $6,88(0,87)$ & $7,1(0,82)$ & $0,327(\mathbf{0 , 2 5})$ \\
\hline
\end{tabular}

Fuente: Elaboración propia

Por lo tanto, aunque no se puede observar un tamaño del efecto significativo (effect size), si miramos la $A E$ y tenemos en cuenta como limitación que la mayoría de los participantes, 14 docentes de un total de 20 , sólo recibieron un total de 15,5 horas de formación, tenemos una variable que se acerca a tener un tamaño del efecto small.

Esto, tal y como indican estudios anteriores (Bisquerra, 2015), deja entrever que, si nos apoyásemos en un futuro en intervenciones de mayor duración, este valor podría ser superior, avalando los hallazgos del análisis temático anteriormente descrito. 


\section{Conclusiones y Discusión}

El objetivo de este estudio es analizar los cambios percibidos por un claustro de secundaria en sus competencias emocionales tras haber realizado una intervención integral de coaching, así como las percepciones de otros miembros de la comunidad educativa.

Los resultados cualitativos diferenciaron 6 dimensiones de cambios. Entre estas, la principal mencionada por todos los colectivos implicados fue la mejora general de las competencias emocionales en docentes, avalada también por los análisis cuantitativos, que aunque con valores no significativos, se pueden comparar a los obtenidos en programas similares (Gutiérrez-Lestón et al., 2018).

Las otras dimensiones percibidas como cambios fueron distintas según los colectivos que intervinieron y coincidieron con otros estudios. Entre ellas, una gestión del aula más práctica y una mayor motivación del alumnado, desde el punto de vista de los estudiantes (Giner y Pérez, 2017), y un grupo de cambios, que se ha visto que corresponden con la secuencia de los 4 niveles de evaluación de la formación, propuestos por Kirkpatrick (2007), según la opinión de los mismos docentes (Pérez-Escoda et al., 2013; García-Navarro, 2017). Se identificaron dos últimas dimensiones en los profesores y en el equipo directivo: los facilitadores de la percepción del cambio, como la participación y el generar un espacio de reflexión dentro y fuera del aula; y otros que la dificultaban (Pérez-Escoda et al., 2013), como la falta de tiempo para profundizar (Obiols y Giner, 2011; Bisquerra, 2015) y el solape con programa similares.

Nuestro estudio evidencia que, a mayor cercanía e interacción con los docentes, mayor fue la sensibilización y percepción de mejora también en las competencias emocionales de los otros miembros de la comunidad educativa, hablando de un posible efecto "contagio" y que, como citan estudios similares (Garcia-Navarro, 2017), si todo el equipo directivo no participa activamente en el programa, esta percepción de cambio puede verse muy limitada, así como la continuidad de la intervención.

En estudios anteriores (Pérez-Escoda et al., 2013) se notó que formaciones de 30 horas no habían sido suficientes para cambiar pensamientos y observar efectos duraderos en el desarrollo de las competencias emocionales, aunque sí habían facilitado concienciarse de la necesidad de un cambio, que para ser sostenible en el tiempo, parecía necesitar de programas de mayor duración (Bisquerra, 2015), de la elaboración de un plan de acción y de un seguimiento realizado por parte de un profesional (Obiols y Giner, 2011). Una importante contribución de nuestro estudio consiste en afirmar que, en el programa íntegro realizado con el grupo piloto, en algunos de sus participantes, sí se ha percibido una modificación de conductas, gracias a la introducción de estos elementos, a pesar de ser de sólo 25 horas.

Interpretamos que una intervención integral en coaching como la nuestra sirve para potenciar el desarrollo de las competencias emocionales, algo que, con objetivos distintos, también corroboran otros estudios recientes (Díaz et al., 2019; Huertas y Romero, 2019).

Por lo tanto, el uso del coaching en educación parece mejorar, en general, la competencia del docente-coach, que en nuestro estudio, como en anteriores (Albaladejo, 2010), coinciden con las competencias emocionales, realizando además, en particular, una mejora en las relaciones y convivencia (función tutorial), con un impacto favorable sobre el clima de la comunidad escolar (Pérez-Escoda et al., 2013), permitiendo este crecimiento emocional que facilita el desarrollo integral de las personas (Pérez-Escoda et al., 2013; Riquelme, 2012).

Una de las mayores limitaciones de nuestro estudio es que para llevar a cabo programas de este estilo se necesita el compromiso de todo el centro educativo y debido a que no todo el equipo directivo participó en la intervención, no le hemos podido dar continuidad, pudiendo haber sido prematuro querer abarcar las percepciones de cambio de los varios colectivos y no dando margen a que se incorporen posibles aprendizajes, a pesar de tener a la mayoría de implicados muy 
interesados en hacerlo. A esto se añade que, siendo la nuestra una intervención contextualizada, no hemos podido eliminar o modificar lo que ya se estaba realizando en el centro sobre temáticas similares y, por lo tanto, integrarlo con nuestro programa.

Como prospectiva de nuestro estudio se desprende que para llevar a cabo programas de tal envergadura se necesita de una importante visión estratégica del centro e inversión económica, contando con más recursos humanos y una intervención más dilatada en el tiempo.

En definitiva, nuestros resultados apuntarían a que estos programas de coaching en educación ayudan al docente a generar este "pensamiento reflexivo" (Schön y Bayo, 1998), tanto sobre el desarrollo de sus competencias emocionales, así como sobre su nuevo rol, de naturaleza más bien tutorial y de orientación, facilitando el buen aprendizaje para el alumnado y animando a seguir en esta línea de investigación de cara al futuro.

\section{Referencias bibliográficas}

Akiba, M. y Wilkinson, B. (2016). Adopting an International Innovation for Teacher Professional Development: State and District Approaches to Lesson Study in Florida. Journal of Teacher Education, 67(1), 74-93. https://doi.org/10.1177/0022487115593603

Albaladejo, M. (2010). Coaching y competencias emocionales. Padres y Maestros / Journal of Parents and 10-14. https://revistas.comillas.edu/index.php/padresymaestros/article/view/1195

Averill, R., Drake, M., Anderson, D. y Anthony, G. (2016). The use of questions within in-themoment coaching in initial mathematics teacher education: enhancing participation, reflection, and co-construction in rehearsals of practice. Asia-Pacific Journal of Teacher Education, 44(5), 486-503. https://doi.org/10.1080/1359866X.2016.1169503

Bécart, A. y Ramírez Garrido, J.D. (2016). Fundamentos del coaching educativo: caracterización, aplicaciones y beneficios desde los cuatro pilares del saber. Plumilla educativa, 18, 344-361.

Bengo, P. (2016). Secondary mathematics coaching: The components of effective mathematics coaching and implications. Teaching and Teacher Education, 60, 88-96. https://files.eric.ed.gov/fulltext/ED573236.pdf

Bisquerra, R. (2003). Educación emocional y competencias básicas para la vida. Revista de Investigación Educativa, 21(1), 7-43. https://revistas.um.es/rie/article/view/99071

Bisquerra, R. (2015). Psicopedagogía de las emociones. Editorial Síntesis.

Bisquerra, R. y Pérez-Escoda, N. (2007). Las competencias emocionales. Educación XX1, 10, 6182. https://doi.org/10.5944/educxx1.1.10.297

Braun, V., y Clarke, V. (2006). Using thematic analysis in psychology. Qualitative Research in Psychology, 3(2), 77-101. https://doi.org/10.1191/1478088706qp063oa

Creswell, J.W. y Plano Clark, V. L. (2011). Designing and Conducting, Mixed Methods Research. SAGE Publications. 
Day, C., Gu, Q. y Sammons, P. (2016). The impact of leadership on student outcomes: how successful school leaders use transformational and instructional strategies to make a difference. Educational Administration Quarterly, 52(2), 221-258. https://doi.org/10.1177 / $\underline{0013161 X 15616863}$

Díaz, O., Cortés, A. y Serra, A. (2019). ¿Hacia un modelo integral de coaching educativo?: Inferencia hacia competencias ejecutivas y liderazgo. Revista Iberoamericana de Psicología, 12(3), 101-112. http://orcid.org/0000-0001-6836-6816

Fernández-Enguita, M. (2016). La educación en la encrucijada. Fundación Santillana.

García Navarro, E. (2017). Formación del profesorado en educación emocional: Diseño, aplicación y evaluación. [Tesis doctoral, Universidad de Barcelona].

Giner, A. y Pérez, G. (2017). Coaching educativo y mejora de las competencias emocionales. PsiAra. Colegio Oficial de Psicología de Catalunya.

Goleman, D. (1996). Inteligencia emocional. Kairos.

Grant, A. M. (2019). Solution-focused coaching: The basics for advanced practitioners. The Coaching Psychologist, 15(2), 44-53. https://iiabc.org/wp-content/uploads/2019/10/solutionfocused-coaching-pages-75-88.pdf

Gutiérrez-Lestón, C., Eroles, M., Pérez-Escoda, N. y Reguant, M. (2018). Una experiència d'educació emocional al casal d'estiu de la Granja Escola: una innovació a l'àmbit de l'oci educatiu. Comunicación presentada en las XIV Jornadas de Educación Emocional. La educación emocional en el centro del cambio educativo, 164-174. Barcelona, España.

Hopkins W.G., Marshall S.W., Batterham A.M. y Hanin J. (2009). Progressive statistics for studies in sports medicine and exercise science. Med Sci Sports Exerc. 41(1)312. https://doi.org/10.1249/MSS. 0b013e31818cb278

Huertas-Fernández, J. y Romero-Rodríguez, S. (2019). La autonomía emocional en el profesorado de secundaria. Análisis en el marco de un proceso de coaching personal. Revista Española de Orientación y Psicopedagogía, 30(3), 120-139.

https://doi.org/10.5944/reop.vol.30.num.3.2019.26276

Jiménez, R. (2012). La investigación sobre coaching en formación del profesorado: una revisión de estudios que impactan en la conciencia sobre la práctica docente. Profesorado. Revista de curriculum y formación del profesorado, 16(1), 238-252.

https://www.redalyc.org/pdf/567/56724377014.pdf

Kirkpatrick, D. L. y Kirkpatrick, J. D. (2006). Evaluación de acciones formativas: los cuatro niveles. Epise. Gestión 2000.

Lenhard, W. y Lenhard, A. (2016). Calculation of Effect Sizes. Psychometrica. https://doi.org/ $\underline{10.13140 / R G \cdot 2.2 .17823 .92329,}$

López, J., Sánchez, M. R., Altopiedi, M. y Oliva, N. (2018). Formación de directivos escolares mediante un programa basado en el coaching grupal, la retroalimentación y el análisis de la práctica. Profesorado: Revista de Currículum y Formación del Profesorado, 22(1), 481-500. https://recyt.fecyt.es/index.php/profesorado/article/view/63654

Maestre, M.M., Nail, O. y Rodríguez-Hidalgo, A.J. (2017). Desarrollo de competencias TIC y para 
la educación inclusiva en la formación inicial práctica del profesorado. Bordón, 69(3), 1-16.

Mora, F. (2017). Neuroeducación: sólo se puede aprender aquello que se ama. Alianza Editorial.

Nelsen, J., Lott, L. y Glenn, H. S. (2013). Positive discipline in the classroom : developing mutual respect, cooperation, and responsibility in your classroom. Three Rivers Press.

Obiols, M. y Giner, A. (2011). El modelo educativo de Bolonia y competencias docentes. Aportaciones desde el coaching educativo. Univest 2011. Universidad.

OCDE. (2016). Low-performing students why they fall behind and how to help them succeed. OECD Publishing. https://www.oecd-ilibrary.org/education/low-performing-students 9789264250246-en.htm

Ozmen, O. (2019). A Scale Development Study to Measure Secondary School Teachers' Opinions On Coaching Behaviours. Eurasian Journal of Educational Research, 19(79), 133-166. https://dergipark.org.tr/en/pub/ejer/issue/42986/520748

Pérez-Escoda, N., Filella, G., Soldevila, A. y Fondevila, A. (2013). Evaluación de un programa de educación emocional para profesorado de primaria. Educación XX1, 16(1), 233-253. https://www.redalyc.org/articulo.oa?id=70625886012

Pérez-Escoda, N., Berlanga, V. y Alegre, A. (2019). Desarrollo de competencias socioemocionales en educacón superior: evaluación del postgrado en educación emocional. Bordón, 71(1), 97113. https://doi.org/10.13042/Bordon.2019.64128

Pérez-Escoda, N., Bisquerra, R., Filella, G. y Soldevila, A. (2010). Construcción del cuestionario de desarrollo emocional de adultos (QDE-A). Revista Española de Orientación y Psicopedagogía, 21(2), 367-379. https://doi.org/10.5944/reop.vol.21.num.2.2010.11539

Pertegal, M. L. (2011). Competencias socioemocionales en el desarrollo profesional del maestro. Educación XX1, 14(2), 237-260. https://doi.org/10.5944/educxx1.14.2.253

Poblete, M. (2006). Las competencias, instrumento para un cambio de paradigma. Investigación en educación matemática. Actas del X Simposio de la Sociedad Española de Investigación en Educación Matemática. Huesca, 6-9 de septiembre de 2006.

Rakap, S. (2017). Impact of Coaching on Preservice Teachers' use of Embedded Instruction in Inclusive Preschool Classrooms. Journal of Teacher Education, 68(2), 125-139. https://doi.org/10.1177/0022487116685753

Riquelme, C. J. (2012). Las competencias docentes del profesor de formación y orientación laboral en el marco de la formación profesional. Las competencias básicas. Competencias profesionales del docente. Universidad de Castilla-La Mancha, (2), 501-518.

Rodríguez-Hidalgo, A. J., Calmaestra, J. y Maestre, M. (2015). Desarrollo de competencias en el Practicum de maestros: ABP y Coaching Multidimensional. Revista de Currículum y Formación del Profesorado, 19(1), 414-434.

https://www.redalyc.org/articulo.oa?id=56738729025

Rosa, G., Riberas, G., Navarro-Segura, L. y Vilar, J. (2015). El coaching como herramienta de trabajo de la competencia emocional en la formación de estudiantes de educación social y trabajo social de la Universidad Ramón Llul, España. Formación universitaria, 8(5),77-90. https://www.redalyc.org/articulo.oa?id=373544192009

Ruzek, E. A., Hafen, C. A., Allen, J. P., Gregory, A., Mikami, A. Y. y Pianta, R. C. (2016). How 
teacher emotional support motivates students: The mediating roles of perceived peer relatedness, autonomy support, and competence. Learning and instruction, 42, 95-103. https://doi.org/10.1016/ i.learninstruc.2016.01.004

Salavert, R. (2015). Coaching: An Apprenticeship Approach for the 21st Century. International Journal of Educational Leadership and Management, 3(1), 4-24. https://doi.org/10.4471/ijelm.2015.02

Sánchez, B. y Boronat, J. (2014). Coaching Educativo: Modelo para el desarrollo de competencias intra e interpersonales. Educación XX1,17(1), 221-242.

https://doi.org/10.5944/educxx1.17.1.10712

Sandín Esteban, M. P. (2003). Investigación cualitativa en educación : fundamentos y tradiciones. McGraw-Hill.

Schön, D. A. y Bayo, J. (1998). El profesional reflexivo: cómo piensan los profesionales cuando actúan. Paidós.

Showers, B. (1984). Peer coaching: A strategy for facilitating transfer of training. Oregon University: Center for Educational Policy and Management.

Torrijos, P. (2016). Desarrollo y evaluación de competencias emocionales para profesores mediante una intervención por programas. (Tesis Doctoral). Universidad de Barcelona.

Torrijos, P., Torrecillas, E. M. y Rodríguez, M. J. (2018). Experimental evaluation of emotional development programmes for teachers in Secondary Education. Anales de Psicologia, 34(1) 68-76. https://dx.doi.org/10.6018/analesps.34.1.273451.

UNESCO. (2016). Escuelas en acción, ciudadanos del mundo para el desarrollo sostenible: guía para el profesorado - UNESCO Biblioteca Digital. https://unesdoc.unesco.org/ark:/48223/pf0000249129

Valderrama, B. (2015). Fundamentos psicológicos del coaching: un modelo para ser más. EOS.

Wood, C. L. (2016). Role of Professional Development and Multi-Level Coaching in Promoting Evidence-Based Practice in Education. Learning Disabilities: A Contemporary Journal, 14(2), 159. https://files.eric.ed.gov/fulltext/EJ1118436.pdf

Fecha de entrada: 13 marzo 2020

Fecha de revisión: 18 junio 2020

Fecha de aceptación: 7 septiembre 2020 